WS9-P02

The Prospecting Potential of Frequency and Pulse CSEM

\author{
P. Barsukov (Institute of Physics of the Earth RAS) \& E. Fainberg* (Institute \\ of Physics of the Earth RAS)
}

\title{
SUMMARY
}

The depth of exploration, sensitivity and resolution of two methods of marine electromagnetic soundings using a horizontal electric dipole as a field source are investigated. An inline dipole-dipole setup measuring a horizontal electric field in the frequency domain and the vertical electric field in a pulsed mode (time domain) in the near-field source are analysed. It was found that the sensitivity of the pulse method in shallow water is higher than that of the frequency approach. In water depths exceeding $800 \mathrm{~m}$, the sensitivities of both methods are approximately the same. The horizontal resolution of the pulse method is higher throughout the range of depths studied. A new approach to the inversion of sounding results is demonstrated on a model of the geological section. 


\section{Amsterdam |'14}

\section{Introduction}

A number of publications have analysed the technology and results of marine EM hydrocarbon reservoir exploration methods devoted to frequency-domain soundings with a controlled source (fCSEM - Control Source ElectroMagnetic). In the case of onshore EM soundings, two approaches can be employed: pulse methods operating in the time domain (t-CSEM) and frequency (f-CSEM) methods based on equivalent positions. In this paper, we consider the potential of both methods in two configurations: an inline source-receiver $H E D-E x(f)$ and a pulse $H E D-E z(t)$ setup operating with a horizontal electric dipole (HED) as the source and horizontal (Ex) and vertical (Ez) (Barsukov, Fainberg, 2013) receivers, respectively. The possibilities and limitations of both methods are considered for these two model examples.

As an electrical model of an HC reservoir, we selected a 100-m-thick rectangular plate with a resistivity of $20 \mathrm{Ohm}-\mathrm{m}$. The plate lies in a sediment column with a resistivity of $1 \mathrm{Ohm}-\mathrm{m}$ at a depth of $1 \mathrm{~km}$ or $2 \mathrm{~km} \mathrm{BSF}$. The water column has a resistivity of $0.28 \mathrm{Ohm}-\mathrm{m}$ and a depth of 50 to $1000 \mathrm{~m}$. The HED-Ex(f) and HED-Ez(t) setups are arranged on the seabed. In the case of f-CSEM, the frequency band of $0.01 \leq \mathrm{f} \leq 10 \mathrm{~Hz}$ and offsets of $1 \leq R \leq 10 \mathrm{~km}$ are analysed, with the observation point in the centre of the configuration; in the case of t-CSEM, the time range of $0.1 \leq \mathrm{t} \leq 16 \mathrm{~s}$ is analysed for a fixed offset of $R=1.5 \mathrm{~km}$. A noise level of $10^{-15} \mathrm{~V} /\left(\mathrm{Am}^{2}\right)$ is employed for both configurations. Figures 1-3 demonstrate the results of these investigations.
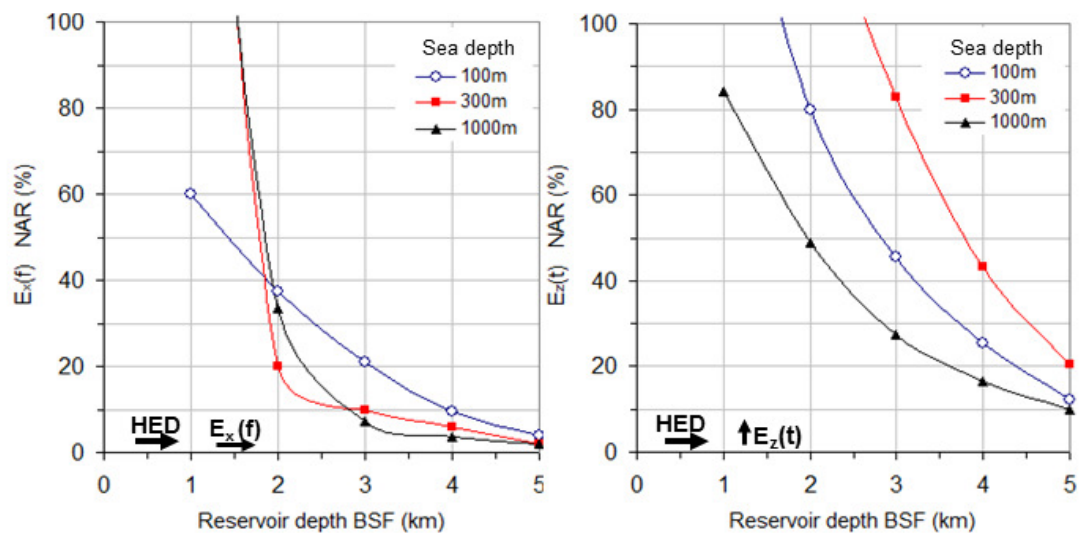

Figure 1 NAR dependence versus depth for a reservoir $5 \times 10 \mathrm{~km}$ in size and $100 \mathrm{~m}$ thick, with a resistivity of $20 \mathrm{Ohm}-\mathrm{m}$.

The graphs in Figure 1 allow us to estimate the maximum possible detection depth of the reservoirs. For example, for NAR $=30 \%$ and a sea depth of $300 \mathrm{~m}$, the maximal detection depth is approximately $2 \mathrm{~km}$ for $H E D-E x(f)$ and $4.5 \mathrm{~km}$ for $H E D-E z(t)$. Here $\mathrm{NAR}(\%)=100\left(\mid \mathrm{E}_{\text {anom }} / \mathrm{E}_{\text {norm }} \mathrm{l}-1\right)$.

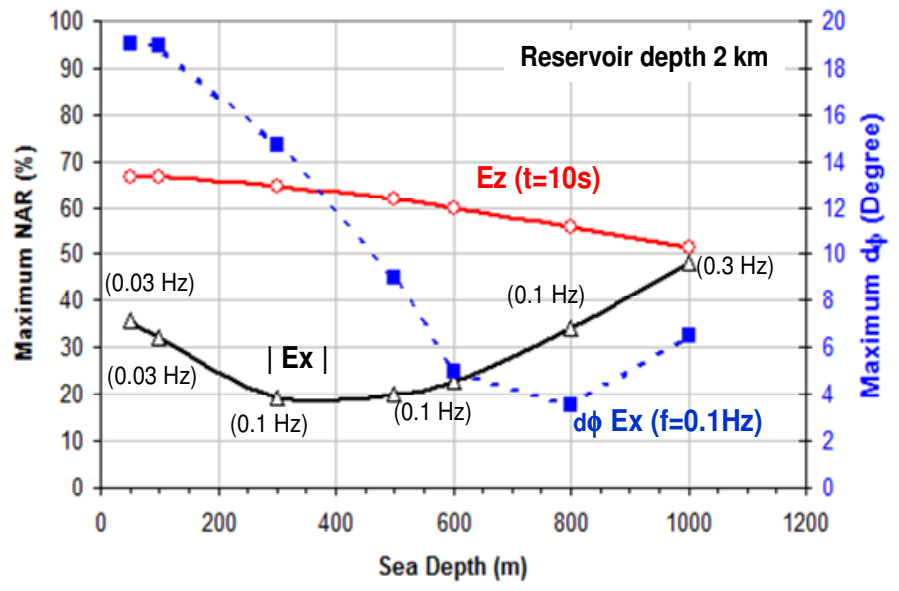

Figure 2 The maximum anomalous effects for the HED-Ex(f) and HED-Ez(t) setups versus sea depth. The reservoir is $5 \times 10 \mathrm{~km}$ in size, with $T=2000 \mathrm{Ohm}-\mathrm{m}^{2}$ and a depth $\mathrm{H}$ of $2 \mathrm{~km} \mathrm{BSF}$. The frequencies corresponding to the maximal effects are shown in brackets. The offset is $6 \mathrm{~km}$ for f-CSEM and $1.5 \mathrm{~km}$ for $t$-CSEM. 


\section{Amsterdam ' 14}

In the time domain, the $E z$ NAR decreases monotonically from $68 \%$ to $50 \%$ as the water depth increases from $50 \mathrm{~m}$ to $1 \mathrm{~km}$. In the frequency domain, the NAR reaches at least $\sim 20 \%$ in the seadepth range of 250 to $500 \mathrm{~m}$, and at greater depths of up to $1 \mathrm{~km}$, the NAR increases and becomes comparable to the NAR for t-CSEM. The phase anomaly of HED-Ex(f) at the optimum frequency of 0.1 $\mathrm{Hz}$ is $\sim 19^{\circ}$ for shallow water but decreases sharply to $\sim 4-8^{\circ}$ for sea depths of more than $600 \mathrm{~m}$.
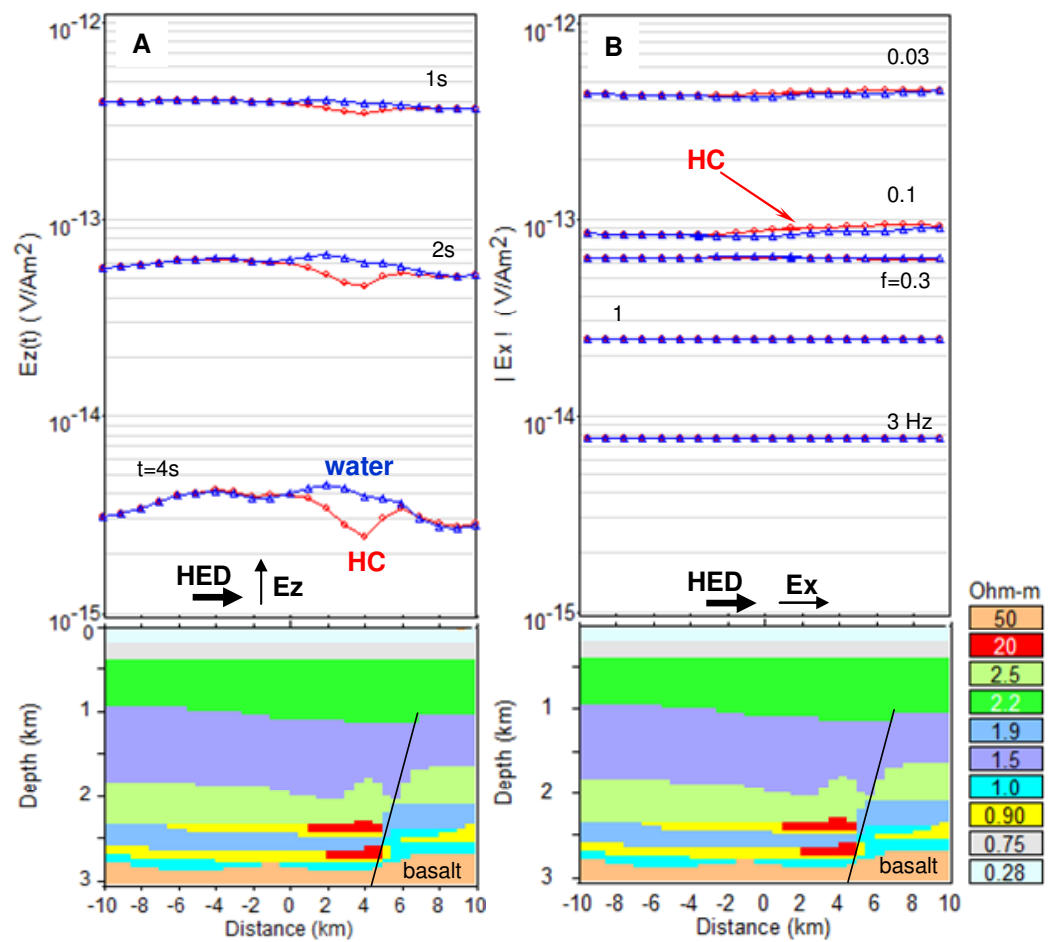

Figure 3 The pulse $(A)$ and frequency $(B)$ responses from reservoirs with "HC" and "water" fluid. The responses are masked by the underlying basaltic layer.

\section{Conclusions}

Based on the modelling results, we can conclude that the most favourable sea depths for the sounding of $\mathrm{HC}$ reservoirs located at a depth of $2 \mathrm{~km}$, when using the $H E D-E x(f)$ configuration, are less than $200 \mathrm{~m}$ or more than $800 \mathrm{~m}$. In the sea-depth range of $200-800 \mathrm{~m}$, the anomalous effects are minimal and do not exceed $30 \%$. Anomalous effects in the $H E D-E z(t)$ configuration are weakly dependent on sea depth and reach $\sim 50-70 \%$. In deep-sea conditions, the relative amplitude of the anomaly and the responses of both configurations are almost identical.

The lateral resolution of $H E D-E z(t)$ with respect to deep-lying reservoirs at shallow and moderate sea depths is higher than that of $H E D-E x(f)$ due to the significant difference in the offsets. For deep-sea conditions, the resolutions for both configurations are approximately the same.

For reservoirs that are identical in size but different in resistivity, the range of burial depths at which indistinguishable responses are obtained within the error of surveying is wider for $H E D-E x(f)$ than for $H E D-E z(t)$.

High-resistivity formations overlying or underlying the reservoir screen the anomalous effects in the fields registered by both configurations, but in the pulse configuration, the anomalies remain relatively high.

For a stable inversion of marine CSEM data, a "seismic skeleton" can be applied based on the geological interpretation of a seismic survey. Inversion in the scope of geoelectric models that contain fixed borders between rock blocks can be achieved only with respect to the resistivities of geological formations.

\section{References}

Barsukov P. and Fainberg E. [2013] A mobile time-domain sounding system for shallow water. First Break, 31 (11), 53-63. 